\title{
EIGENVALUES OF THE LAPLACIAN ON FORMS
}

\author{
JOZEF DODZIUK ${ }^{1}$
}

\begin{abstract}
Some bounds for eigenvalues of the Laplace operator acting on forms on a compact Riemannian manifold are derived. In case of manifolds without boundary we give upper bounds in terms of the curvature, its covariant derivative and the injectivity radius. For a small geodesic ball upper and lower bounds of eigenvalues in terms of bounds of sectional curvature are given.
\end{abstract}

In [2] Cheng proves a beautiful comparison theorem for the first eigenvalue of the Laplacian $\Delta_{0}$ on functions for a geodesic ball in a Riemannian manifold, and derives as a consequence, upper bounds for higher eigenvalues of the Laplacian on functions for a compact manifold. These upper bounds are derived by taking the first eigenfunctions with Dirichlet boundary conditions for small balls, extending them by zero to the whole manifold, and estimating the Rayleigh-Ritz quotient of an appropriate linear combination. The same procedure cannot be applied to forms of positive degree since an eigenfunction for a ball satisfying either absolute or relative boundary conditions will not be in the Sobolev space $H^{1}$ when extended by zero to the whole manifold. In this note a modification of the method of Eichhorn [4] is used to prove that, for certain forms on a ball which vanish on the boundary, it is possible to estimate the Rayleigh-Ritz quotient in terms of geometric quantities. These forms are in $H^{1}$ when extended by zero and Cheng's argument applied to them gives upper bounds for eigenvalues on a closed manifold. Our result is much less elegant than Cheng's theorem. In the first place we have to require that the geodesic ball is contained within the cut locus. Hence, all estimates of higher eigenvalues depend on the injectivity radius. Secondly, Cheng obtained explicit estimates of eigenvalues of the Laplacian $\Delta_{0}$ in terms of geometric quantities (cf. [2, Corollaries $2.2,2.3$, Theorem 2.4]), whereas we only say which geometric quantities determine the bounds of eigenvalues but give no estimates of the actual bounds. Explicit estimates could be derived from our method, but the constants are so complicated that we were unable to obtain any useful information from them. It is an interesting question, whether all of the geometric quantities which appear in our estimates (sectional curvature, injectivity radius, the bounds for the Kern tensor $R^{(1)}$ ) are really necessary. We do not know the answer for closed manifolds. However, in $\S 3$ we show, following a suggestion of J. Cheeger, that for a geodesic ball of radius smaller than the radius of injectivity, eigenvalues of the Laplacian $\Delta_{p}$ can be estimated from above and below in terms of bounds of sectional curvature.

It is a pleasure to thank J. Cheeger for suggestions which led to improvement of this paper.

Received by the editors March 3, 1981 and, in revised form, December 9, 1981.

1980 Mathematics Subject Classification. Primary 58G25; Secondary 35P15.

${ }^{1}$ Partially supported by an NSF Grant and a Sloan Fellowship. This note was written while the author enjoyed the hospitality of the Mathematical Institute at the University of Oxford. 
1. Differential geometric preliminaries. Let $M$ be a $C^{\infty}$ oriented, Riemannian manifold of $n$ dimensions. We will denote by $\langle$,$\rangle the inner products induced by the$ Riemannian metric in fibres of various tensor bundles, and by $\mid$ the corresponding norm. Let $R^{(1)}$ be the tensor introduced by Kern [8]:

$$
R^{(1)}(U, V, X, Y)=\left(\nabla_{U} R\right)(V, X, Y)-\left(\nabla_{X} R\right)(Y, U, V),
$$

where $R$ denotes the Riemann curvature tensor and $\nabla$ is the covariant derivative of the Levi-Civita connection.

DEFINITION 1.2 (CF. [7]). The curvature of $M$ is said to be $(\alpha, \beta, \gamma)$-bounded on a subset $N \subset M$ if the sectional curvature $K$ satisfies $\alpha \leq K \leq \beta$ for every plane section at every point $p \in N$, and if $\left|R^{(1)}(u, u, v, v)\right| \leq \gamma|u||v|$ for every pair of orthogonal vectors $u, v \in T_{p} M$ and every $p \in N$. The following lemma is contained in [5, Lemma 1 and 7, Corollary 1].

To state it we need some notation. For $\nu \in \mathbf{R}$, define

$$
c_{\nu}(t)=\frac{\sin t \sqrt{\nu}}{t \sqrt{\nu}}, \quad t \geq 0 .
$$

It is understood that $C_{0}(t) \equiv 1$; for $\nu<0$ we use the complex extension of sin, $C_{\nu}(0)=1$; and for $\nu>0, C_{\nu}$ is defined only on $[0, \pi / \sqrt{\nu})$.

LEMMA 1.3. Suppose $B(q, \rho) \subset M$ is the geodesic ball of radius $\rho>0$ centered at $q \in M$, and $\overline{B(q, \rho)}$ is a diffeomorphic image of a closed ball of radius $\rho$ centered at $0 \in T_{q} M$. Suppose further that the sectional curvature $K$ satisfies $\alpha \leq K \leq \beta$ on $\overline{B(q, \rho)}$ (in particular $\rho<\pi / \bar{\beta}$ ) for some $\alpha \leq 0 \leq \beta$. Let $r=r(x)$ denote the geodesic distance from $x \in M$ to $q$. Then the components of the metric tensor with respect to a normal geodesic coordinate system centered at $g$ satisfy

$$
C_{\beta}^{2}(r) \sum_{i=1}^{n} \varsigma^{i^{2}} \leq \sum_{i, j} g_{i j}(x) \varsigma^{i} \varsigma^{j} \leq C_{\alpha}^{2}(r) \sum_{i=1}^{n} \varsigma^{i^{2}}
$$

for $\operatorname{all}\left(\varsigma^{1}, \ldots, \zeta^{n}\right) \in \mathbf{Z}^{n}, x \in \overline{B(q, \rho)}$.

In addition, if the curvature is $(\alpha, \beta, \gamma)$-bounded on $\overline{B(q, \rho)}$ for a constant $\gamma>0$, there exists a continuous function $g:[0, \rho] \rightarrow \mathbf{R}$ determined only by $\alpha, \beta$ and $\gamma$ such that

$$
\left|\Gamma_{i j}^{k}(x)\right| \leq g(r), \quad \text { for all } i, j, k=1,2, \ldots, n \text { and } r \leq \rho
$$

2. Rayleigh-Ritz quotient for certain forms on a ball. In this section we exhibit certain differential forms and estimate their Rayleigh-Ritz quotients. Let $f=$ $f_{\alpha, \rho}$ be the first eigenfunction of the Laplacian $\Delta_{0}$ satisfying Dirichlet boundary conditions for the ball of radius $\rho$ in the space of constant curvature $\alpha$. We assume that $f$ is normalized so that its $L^{2}$ norm is equal to one. Let $B=B(q, \rho) \subset M$ be a ball satisfying the assumptions of Lemma 1.3. As observed in [2], $f$ is a function of the geodesic distance from the center and it can be transplanted to $B$ via the exponential map. Let $x^{1}, x^{2}, \ldots, x^{n}$ be a normal geodesic coordinate system centered at $q$. Define a $p$-form, $0<p<n, \omega$ on $\bar{B}$ by

$$
\omega=f(r) \omega_{0}, \quad \omega_{0}=d x^{1} \wedge \cdots \wedge d x^{p} .
$$


The Rayleigh-Ritz quotient of $\omega, R(\omega)$ is

$$
R(\omega)=\frac{(d \omega, d \omega)_{B}+(\delta \omega, \delta \omega)_{B}}{(\omega, \omega)_{B}}
$$

where $\delta$ is the formal adjoint of the exterior derivative $d$ and, for every form $\eta$, $(\eta, \eta)_{B}=\int_{B}|\eta|^{2} d V=\int_{B} \eta \wedge * \eta$. Here $*$ is the Hodge operator and $d V$ denotes the volume element.

Proposition 2.3. Suppose $B(q, \rho)=B \subset M$ satisfies the assumptions of Lemma 1.3 (i.e. $\rho$ is smaller than the injectivity radius and the curvature is $(\alpha, \beta, \gamma)$ bounded on $\bar{B}$ ) and $\omega$ is defined by (2.1). Then $R(\omega) \leq C$, where $C=$ $C(n, p, \alpha, \beta, \gamma, \rho)$ is a constant depending only on $n, p, \alpha, \beta, \gamma$ and $\rho$.

Proof. To estimate $R(\omega)$ from above we will estimate $(\omega, \omega)_{B}$ from below and $(d \omega, d \omega)_{B}+(\delta \omega, \delta \omega)_{B}$ from above. Now

$$
(\omega, \omega)_{B}=\int_{\Sigma\left(x^{i}\right)^{2} \leq \rho^{2}} f^{2}\left|\omega_{0}\right|^{2} \sqrt{g} d x^{1} \cdots d x^{n},
$$

where $g=\operatorname{det}\left(g_{i j}\right)$. Recall also that for an arbitrary form

$$
|\eta|^{2}=g^{i_{1} j_{1}} \cdots g^{i_{p} j_{p}} \eta_{i_{1} \cdots i_{p}, j_{1} \cdots j_{p}}
$$

where $\left(g^{i j}\right)=\left(g_{k l}\right)^{-1}$. Thus it follows from (1.4) that there exists a constant $C_{1}>0$ depending only on $\alpha, \beta, \rho, n, p$ such that $\left|\omega_{0}\right|^{2} \sqrt{g} \geq C_{1}$ for all $x \in B(q, \rho)$. From this

$$
(\omega, \omega)_{B} \geq C_{1} \int_{\Sigma\left(x^{i}\right)^{2} \leq \rho^{2}} f^{2} d x^{1} \cdots d x^{n} .
$$

Now note that (1.4) implies that the volume element $\sqrt{g_{\alpha}} d x^{1} \cdots d x^{n}$ for the metric of constant sectional curvature $\alpha$ satisfies $\left(\sqrt{g_{\alpha}}\right)^{-1} \geq C_{2}$ on a ball of radius $\rho$. The constant $C_{2}$ depends only on $\rho$ and $\alpha$.

Therefore

$$
(\omega, \omega)_{B} \geq C_{1} C_{2} \int_{\Sigma\left(x^{i}\right)^{2} \leq \rho^{2}} f^{2} \sqrt{g_{\alpha}} d x^{1} \cdots d x^{n}=C_{1} C_{2}
$$

since $f$ was a normalized eigenfunction.

To estimate the numerator of $R(\omega)$ note that up to a sign $\delta \omega=* d * \omega$. Therefore we have

$$
\begin{aligned}
|\delta \omega| & =|d * \omega|=\left|f^{\prime} d r \wedge * \omega_{0}+f d * \omega_{0}\right| \leq\left|f^{\prime}\right| \cdot\left|\omega_{0}\right|+|f|\left|\delta \omega_{0}\right| \\
& \leq\left(\left|f^{\prime}\right|^{2}+f^{2}\right)^{1 / 2}\left(\left|\omega_{0}\right|^{2}+\left|\delta \omega_{0}\right|^{2}\right)^{1 / 2} .
\end{aligned}
$$

Moreover,

$$
|d \omega|=\left|f^{\prime} d r \wedge \omega_{0}\right| \leq\left|f^{\prime}\right|\left|\omega_{0}\right| .
$$

To establish the inequalities above we used the fact that * is an isometry and that $d r$ is a unit covector. Using (2.4) and (1.4) we can bound $\left|\omega_{0}\right|$ from above by a constant depending only on $\beta, \rho, n, p$. Moreover, the general formula (cf. [10])

$$
\left(\delta_{\eta}\right)_{i_{1} \cdots i_{p-1}}=-g^{i j}\left(\frac{\partial}{\partial x^{j}} \eta_{i i_{1} \cdots i_{p-1}}-\Gamma_{j i}^{k} \eta_{k i_{1} \cdots i_{p-1}}-\sum_{l=1}^{p-1} \Gamma_{j i_{l}}^{k} \eta_{i i_{i} \cdots i_{l-1} k i_{l+1} \cdots i_{p}}\right)
$$


enables one to estimate $\left|\delta \omega_{0}\right|$ if one can estimate $g^{i j}$ and $\Gamma_{j i}^{k}$. Such estimates follow from Lemma 1.3. Thus it follows from (2.6) and (2.7) that $|d \omega|^{2}+|\delta \omega|^{2} \leq$ $C_{3}\left(\left(f^{\prime}\right)^{2}+f^{2}\right)$, where $C_{3}$ depends only on $\alpha, \beta, \gamma, \rho, n, p$.

It is well known (cf. [1, p. 254]) that $\sqrt{g_{\alpha}} \geq \sqrt{g}$, where $\sqrt{g_{\alpha}} d x^{1} \cdots d x^{n}$ is the volume element on the space of constant sectional curvature $\alpha$, since we are assuming that all sectional curvatures on $B(q, \rho)$ are greater than or equal to $\alpha$. Therefore, using the fact that $f$ is the normalized first eigenfunction we obtain

$$
\begin{aligned}
(d \omega, d \omega)_{B}+(\delta \omega, \delta \omega)_{B} & \leq C_{3} \int_{\Sigma\left(x^{i}\right)^{2}<\rho^{2}}\left(\left(f^{\prime}\right)^{2}+f^{2}\right) \sqrt{g_{\alpha}} d x^{1} \cdots d x^{n} \\
& =C_{3}(\lambda+1),
\end{aligned}
$$

where $\lambda=\lambda(\alpha, \rho)$ is the first eigenvalue of $\Delta_{0}$ on a ball of radius $\rho$ in the $n$ dimensional space of constant sectional curvature $\alpha$. (2.8) and (2.5) prove the proposition.

A similar argument was used by Eichhorn [4] to prove that the spectrum of $\Delta_{p}$ is nondiscrete for complete manifolds with $(\alpha, \beta, \gamma)$-bounded curvature for some $\alpha\langle\beta, \gamma\rangle 0$ and injectivity radius bounded from below.

REMARK 2.9. The proof above shows that for sufficiently small $\rho$

$$
R(\omega) \leq C_{4}(1+\lambda(\alpha, \rho)),
$$

where $C_{4}$ is a constant depending on $n, p, \alpha, \beta, \gamma$ but not on $\rho$. Clearly $\lambda(\alpha, \rho) \sim$ const $/ \rho^{2}$ when $\rho$ approaches 0 .

3. Eigenvalues for a ball. Proposition 2.3 gives an upper bound for $\lambda_{1, p}$, the first eigenvalue of the Laplacian $\Delta_{p}$ on forms satisfying relative boundary conditions for a ball. The test form $\omega$ used there is very special and it should be possible to give a much better estimate. This is the case as was pointed out to the author by J. Cheeger. The same method will yield estimates of higher eigenvalues.

Consider first an oriented Riemannian manifold $N$ with boundary $\partial N$ and the space of $C^{\infty}$ forms on $N$ satisfying absolute boundary conditions $\omega_{\text {norm }}=$ $d \omega_{\text {norm }}=0$ on $\partial N$ (cf. [9]). The knowledge of the spectra of $\Delta_{p}, 0 \leq p \leq n=$ $\operatorname{dim} N$, is equivalent to knowing the spectra of $\Delta_{p}, 0<p \leq n$, on the space of exact forms with absolute boundary conditions. Actually, this yields information about spectra for forms with relative boundary conditions as well, since * operator commutes with the Laplacian and interchanges absolute and relative boundary conditions.

Proposition 3.1. The spectrum $0<\mu_{1, p}<\mu_{2, p}<\cdots$ of the Laplacian $\Delta_{p}$, $0<p \leq n$, on exact forms of degree $p$ with absolute boundary conditions can be computed as follows:

$$
\mu_{i, p}=\inf _{V} \sup _{\eta \in V \backslash\{0\}}\left\{\frac{(\eta, \eta)}{(\theta, \theta)} \mid d \theta=\omega\right\},
$$

where $V$ ranges over all subspaces of $i$ dimensions of the space of all $C^{\infty}$ exact forms of degree $p$ on $N$.

The proof is rather standard and will be omitted. The main point is to observe that the critical points for the functional

$$
\eta \rightarrow \frac{(d \eta, d \eta)}{(\eta, \eta)}
$$


automatically satisfy the boundary condition $d \omega_{\text {norm }}=0$. If $\eta_{0}$ is such a critical point, then $d \eta_{0}$ is an exact eigenform.

Suppose $g$ and $g^{\prime}$ are two metrics on $N$ such that

$$
\sigma g \leq g^{\prime} \leq \tau g
$$

for some constants $\sigma, \tau>0$. The proposition above allows us to estimate the eigenvalues of the Laplacian for the metric $g^{\prime}$ in terms of those for $g$.

Proposition 3.3. Suppose the metrics $g$ and $g^{\prime}$ on $N$ satisfy (3.2). Let $\left\{\mu_{i, p}\right\}_{i=1}^{\infty}$ (respectively $\left\{\mu_{i, p}^{\prime}\right\}_{i=1}^{\infty}$ ) be the sequence of eigenvalues for exact $p$-form with absolute boundary conditions for the metric $g$ (respectively $\left.g^{\prime}\right)$. Then

$$
\frac{1}{\sigma}\left(\frac{\sigma}{\tau}\right)^{n / 2+p} \mu_{i, p} \leq \mu_{i, p}^{\prime} \leq \frac{1}{\tau}\left(\frac{\tau}{\sigma}\right)^{n / 2+p} \mu_{i, p}
$$

ProOF. The space of the test forms in Proposition 3.1 is independent of the metric. Let $(\eta, \eta)^{\prime}$ be the square of the $L^{2}$ norm of the form $\eta$ for the metric $g^{\prime}$. An easy calculation shows that, if $\eta$ has degree $q$,

$$
\tau^{-q} \sigma^{n / 2}(\eta, \eta) \leq(\eta, \eta)^{\prime} \leq \sigma^{-q} \tau^{n / 2}(\eta, \eta) .
$$

As a consequence, if $Q(\eta)=\sup \{(\eta, \eta) /(\theta, \theta) \mid d \theta=\eta\}$ and $\operatorname{deg} \eta=p$,

$$
\frac{1}{\theta}\left(\frac{\sigma}{\tau}\right)^{n / 2+p} Q(\omega) \leq Q^{\prime}(\omega) \leq \frac{1}{\tau}\left(\frac{\tau}{\sigma}\right)^{n / 2+p} Q(\omega)
$$

with the obvious definition of $Q^{\prime}$. (3.4) follows from this and Proposition 3.1.

Now consider a geodesic ball $B=B(q, \rho)$ with $\rho$ smaller than the injectivity radius at $q$. Assume that the sectional curvature $K$ satisfies $\alpha \leq K \leq \beta$ on $\bar{B}$ for some $\alpha \leq 0 \leq \beta$ (in particular $\rho<\pi / \sqrt{\beta}$ ). Then, by (1.4), the metric on $\bar{B}$ is uniformly bounded from above and below by multiples of the Euclidean metric and, applying Proposition 3.3 we obtain

PROPOSITION 3.5. Let $\left\{\nu_{i, p}(\rho)\right\}_{i=1}^{\infty}$ be the sequence of eigenvalues for exact forms of degree $p$ with absolute boundary conditions for a ball of radius $\rho$ in the Euclidean space. If $B$ is as above and $\left\{\mu_{i, p}\right\}_{i=1}^{\infty}$ are corresponding eigenvalues for $B$, then

$$
C_{\alpha}^{-2}(\rho) \cdot\left(\frac{C_{\alpha}(\rho)}{C_{\beta}(\rho)}\right)^{n+2 p} \nu_{i, p}(\rho) \leq \mu_{i, p} \leq C_{\beta}^{-2}(\rho)\left(\frac{C_{\beta}(\rho)}{C_{\alpha}(\rho)}\right)^{n+2 p} \nu_{i, p}(\rho)
$$

4. Closed manifolds. In this section $M$ is $C^{\infty}$ oriented, Riemannian and compact. We observe that the method of Cheng [2] can be used to estimate higher eigenvalues of $\Delta_{p}$ on a closed manifold $M$ in terms of bounds for $R(\omega)$ for forms constructed in $\S 2$. We recall the minimun characterization of eigenvalues of $\Delta_{p}$ which follows from Courant's min-max principle [3]. Suppose $\lambda_{1, p} \leq \lambda_{2, p} \leq \cdots$ is the sequence of eigenvalues of $\Delta_{p}$, each repeated according to its multiplicity. Let $\left\{\varphi_{i}\right\}_{i=1}^{\infty}$ be the corresponding complete orthonormal sequence of eigenforms. Then

$$
\lambda_{m+1, p}=\inf _{\varphi} \frac{(d \varphi, d \varphi)+(\delta \varphi, \delta \varphi)}{(\varphi, \varphi)}
$$


where $\varphi$ ranges over the set of all $L^{2}$ forms of degree $p$ such that $d \varphi$ and $\delta \varphi$ are in $L^{2}$ and $\varphi$ is orthogonal to $\varphi_{1}, \varphi_{2}, \ldots, \varphi_{m}$. Here the inner product for forms is given by $(\omega, \eta)=\int_{M} \omega \wedge * \eta$ and $d \varphi$ and $\delta \varphi$ are taken in the sense of distributions.

Let $D$ be a subdomain of $M$ with a smooth boundary, and let $\eta$ be a $C^{1}$ form on $\bar{D}$ which vanishes on $\partial D$. Extend $\eta$ to be zero outside $D$. It is easy to see that for the resulting continuous form $\tilde{\eta}$ on $M d \tilde{\eta}$ and $\delta \tilde{\eta}$ are in $L^{2}$ and are in fact represented by the trivial extension by zero of $d \eta$ and $\delta \eta$ computed on $D$. Therefore the forms $\omega$ constructed in $\S 2$ can be used for eigenvalue estimates on $M$.

Having made this observation we can apply the method of proof of Theorem 2.1 of [2] to obtain an upper bound of higher eigenvalues. We need an easy lemma whose proof can be found in $\S 5$ of [6].

LEMMA 4.1. Suppose $M$ is compact, $\operatorname{dim} M=n$ and the Ricci curvature of $M$ satisfies $\operatorname{Ric}_{M} \geq(n-1) \alpha$. Let $N(\epsilon)$ denote the maximal number of disjoint balls of radius $\epsilon$ in $M$. Then

$$
N(\epsilon) \geq \frac{V(M)}{V(2 \epsilon, \alpha)}
$$

where $V(M)$ is the volume of $M$ and $V(2 \epsilon, \alpha)$ denotes the volume of ball of radius $2 \epsilon$ in the space of constant sectional curvature $\alpha$. If $\alpha \leq 0$, and $\epsilon$ is sufficiently small, e.g. $\epsilon \leq 1$,

$$
N(\epsilon) \geq \frac{V(M)}{d \epsilon^{n}}
$$

where $d=d(\alpha, n)$.

Our bound of higher eigenvalues is given in the following theorem.

THEOREM 4.2. Let $M$ be a $C^{\infty}$ oriented, compact, Riemannian manifold. Suppose the curvature of $M$ is $(\alpha, \beta, \gamma)$ bounded, $\alpha \leq 0 \leq \beta$. Let $i_{M}$ denote the injectivity radius of $M$. The $m$ th eigenvalue $\lambda_{m, p}(M)$ of the Laplacian $\Delta_{p}$ on forms of degree $p, 0<p<n$, can be bounded above by a constant depending on $p, n=$ $\operatorname{dim} M, \alpha, \beta, \gamma, V(M)$ and $i_{M}$. More precisely, if $C$ is the constant in Proposition 2.3 , then

$$
\lambda_{m, p}(M) \leq C\left(n, p, \alpha, \beta, \gamma, \min \left(\rho, \frac{1}{2}\left(\frac{V(M)}{d m}\right)^{1 / n}\right)\right),
$$

where $\rho$ is any number in $\left(0, i_{M}\right)$ and $d=d(\alpha, n)$ is the constant appearing in Lemma 4.1.

Proof. We are assuming that $\alpha \leq 0 \leq \beta$. By Lemma 4.1, we can find $m$ disjoint balls in $M$ of radius $\frac{1}{2}(V(M) / d m)^{1 / n}$. The theorem follows by applying the method of proof of Cheng's theorem (Theorem 2.1 of [2]) verbatim.

It follows from Remark 2.8 and the theorem above that

$$
\limsup _{m \rightarrow \infty} \frac{\lambda_{m, p}}{m^{2 / n}} \leq C_{5}
$$

where $C_{5}$ depends on $n, p, \alpha, \beta, \gamma$, and $V(M)$. This is of correct order in view of $\mathrm{H}$. Weyl's asymptotic formula. 


\section{REFERENCES}

1. R. Bishop and R. Crittenden, Geometry of manifolds, Academic Press, New York, 1964.

2. S. Y. Cheng, Eigenvalue comparison theorems and its geometric applications, Math. Z. 143 (1975), 289-297.

3. R. Courant and D. Hilbert, Methods of mathematical physics, vol. 1, Interscience, New York, 1953.

4. J. Eichhorn, Das Spektrum von $\Delta_{p}$ auf offenen Riemannschen Mannigfaltigkeiten mit beschränkter Schmitt-krümmung und beschranktem Kern-Tensor, preprint.

5. S. Hildebrandt, H. Kaul and K. O. Widman, An existence theorem for harmonic mappings of Riemannian manifolds, Acta Math. 138 (1977), 1-16.

6. M. Gromov, Paul Lévy's isoperimetric inequality, preprint.

7. H. Kaul, Schranken für die Christoffelsymbol, Manuscripta Math. 19 (1976), 261-273.

8. J. Kern, Das Pinchingproblem in fastriemannschen Finslerschen Mannigfaltigkeiten, Mansuscripta Math. 4 (1971), 341-350.

9. D. Ray and I. M. Singer, R-torsion and the Laplacian on Riemannian manifolds, Adv. in Math. 7 (1971), 145-210.

10. G. de Rham, Variétés différentiables, Hermann, Paris, 1955.

Department of Mathematics, CUNY, Queens College, Flushing, New York 11367 